\title{
Mechanism of Carrier Photoexcitation in Semiconducting Polymers: The Role of Electron Photoemission in "Photoconductivity" Measurements
}

\author{
Daniel Moses ${ }^{1 \# \text {, Paulo B. Miranda }}{ }^{2}$, Cesare Soci ${ }^{3}$, and Alan J. Heeger ${ }^{1}$ \\ ${ }^{1}$ Institute for Polymers and Organic Solids, University of California at Santa Barbara, Santa \\ Barbara, CA 93106 USA \\ ${ }^{2}$ Departamento de Física, Universidade Estadual Paulista, Caixa Postal 473, Bauru - SP 17015 - \\ 970, Brazil \\ ${ }^{3}$ Current Address INFM - Phys. Deptment, University of Pavia, Italy \\ ${ }^{\#}$ Corresponding author, E-mail: moses@ipos.ucsb.edu;
}

\begin{abstract}
Ultrafast photoinduced absorption by infrared-active vibrational modes (IRAV) is used to detect charged photo-excitations (polarons) in solid films of conjugated luminescent polymers. Experiments, carried out in zero applied electric field, show that polarons are generated within 100 fs with quantum efficiencies of approximately $10 \%$. The ultrafast photoinduced IRAV Absorption, the weak pump-wavelength dependence, and the linear dependence of charge density on pump intensity indicate that both charged polarons and neutral excitons are independently generated even at the earliest times. Measurements of the excitation profile of the transient and steady-state photoconductivity of poly(phenylene vinylene) and its soluble derivatives over a wide spectral range up to $h v=6.2 \mathrm{eV}$ indicate an apparent increase in the "photoconductivity" at hv $>3-4 \mathrm{eV}$ that arises from external currents generated by electron photoemission (PE). After quenching the $\mathrm{PE}$ by addition of $\mathrm{CO}_{2}+\mathrm{SF}_{6}(90 \%: 10 \%)$ into the sample chamber, the bulk photoconductivity is nearly independent of photon energy in all polymers studied, in a good agreement with the IRAV spectra. The single threshold for photoconductivity is spectrally close to the onset of $\pi-\pi^{*}$ absorption, behavior that is inconsistent with a large exciton binding energy.
\end{abstract}

\section{Photoinduced IRAV: Ultrafast Photo-generation of Charged Polarons}

Semiconducting (conjugated) polymers have been known for over twenty years. Research on these materials has intensified in the last decade as a result of the increasing number of applications, including light-emitting diodes, lasers and solar cells [1]. Despite many advances, the nature of the elementary excitations in semiconducting polymers remains controversial [2]. Issues of great importance to the understanding and improvement of polymer devices, such as the exciton binding energy and charge carrier generation mechanism, remain unresolved. Here we use a unique alloptical technique [3,4] based on photoinduced infrared-active vibrational (IRAV) modes to study the photogeneration and recombination dynamics of charged polarons in poly(paraphenylenevinylene), PPV, and its derivatives.

There are two dominant theoretical views of the charge generation mechanism in conjugated polymers [2,5]. The first is based on the model of Su, Schrieffer and Heeger ( $\mathrm{SSH}$ ) [6], which treats the polymer chain as a tight-binding one-dimensional semiconductor in the one-electron approximation and explicitly includes the electron-phonon interaction. The SSH approach assumes that the electron-electron (el-el) interactions are relatively weak because of screening, leading to small exciton binding energies $(\approx 0.1 \mathrm{eV})$ [7]. The coincidence of the onset of absorption and photoconductivity has been used as support for this approach, where charges are directly 
photogenerated. In the second theoretical approach, the el-el interactions are assumed to be dominant so that bound excitons with relatively large binding energy $(\geq 0.4 \mathrm{eV})$ are the elementary photoexcitations. Charged polarons are then indirectly generated by a variety of mechanisms. Studies of excitations generated at high photon energies relative to the onset of $\pi-\pi^{*}$ absorption are particularly important for resolving these issues. Therefore, a direct measurement of the dependence of the efficiency for charge generation $\phi_{c h}$ on the excitation wavelength is particularly important for clarification of the charge generation mechanism.

Recently, we determined the excitation profile of the transient and steady-state photoconductivity (PC) of poly(phenylene vinylene) and its soluble derivatives over a wide spectral range up to $\mathrm{hv}=6.2 \mathrm{eV}$ [8]. We find an apparent increase in the "photoconductivity" at hv $>3-4$ $\mathrm{eV}$ that arises from external currents generated by electron photoemission (PE). However, after quenching the $\mathrm{PE}$ by addition of $\mathrm{CO}_{2}+\mathrm{SF}_{6}$ (90\%:10\%) into the sample chamber, the bulk photoconductivity is found nearly independent of photon energy in all polymers studied, in good agreement with the IRAV spectra.

We have used ultrafast photoinduced IRAV absorption, an all-optical technique with subpicosecond time resolution carried out in zero applied electric field, to investigate the charge generation mechanism in PPV and its soluble derivatives.

The IRAV absorption results from Raman-active vibrational modes that become infrared-active when the local symmetry is broken by self-localization of charges (polaron formation). The IRAV modes have a 1:1 correspondence with the strongest modes observed in on-resonant Raman scattering and have an unusually high infrared absorption cross-section $\left(\sigma_{\text {IRAV }} \sim 10^{-16} \mathrm{~cm}^{2}\right.$, comparable to electronic transitions) [1,6,9]. They are a well-known probe of photo- or dopinginduced charged excitations (solitons, polarons and bipolarons) in conjugated polymers and have been described in detail elsewhere $[1,6,9,10]$. Thus, photoinduced IRAV absorption can address the question of whether charged polarons are photo-generated at ultrafast time scales in conjugated polymers. There is, however, an important distinction between the charges detected by IRAV absorption and by PC. While in the latter charges must be mobile, IRAV absorption is also sensitive to charges in localized states that do not contribute to PC.

The samples were freestanding films with thickness from 1 to $40 \mu \mathrm{m}$. We have studied stretchaligned PPV (draw-ratio $1 / 1_{0}=4$ ) and disordered films of PPV derivatives, such as MEH-PPV (poly[2-methoxy-5-(2'-ethyl)hexyloxy-1,4-phenylene vinylene]) and BuEH-PPV (poly[2-butyl-5(2'-ethyl)hexyl-1,4-phenylene vinylene]), prepared by multiple spin casting from $1 \% \mathrm{w} / \mathrm{v}$ toluene solutions onto sapphire substrates, and subsequently removed from the substrate. For comparison and quantitative determination of the quantum efficiency, we used the $\mathrm{MEH}-\mathrm{PPV} / \mathrm{C}_{60}$ blend $(50 \%$ w/w of the $\mathrm{C}_{60}$ derivative 1-(3-methoxycarbonyl)propyl-1-phenyl[6,6], $\mathrm{C}_{61}$ ).

The optical setup consisted of an amplified Ti:Sapphire system (wavelength $795 \mathrm{~nm}, 1$ $\mathrm{mJ} /$ pulse, $\sim 100 \mathrm{fs}$ pulse duration, $1 \mathrm{kHz}$ repetition rate) pumping two optical parametric amplifiers (OPAs). The first OPA was used to pump the samples and generated pulses tunable between 600 $\mathrm{nm}$ to $250 \mathrm{~nm}$ by nonlinear mixing of pump, signal and idler beams. Additional pump wavelengths were obtained by third- and fourth-harmonic generation of the fundamental wavelength. The second OPA was equipped with a difference-frequency generation stage and generated the probe beam, tunable from 3 to $10 \mu \mathrm{m}$ (1000 to $3300 \mathrm{~cm}^{-1}$ ). The pump and probe pulses overlapped on the sample and the delay between them was controlled with a variable delay line. Polarizations were linear and parallel, with the IR polarized along the stretch direction of the PPV sample. The differential changes in transmission $\left(\Delta \mathrm{T} / \mathrm{T}=\left(\mathrm{T}_{\text {on }}-\mathrm{T}_{\text {off }}\right) / \mathrm{T}_{\text {off }}\right)$ of the probe induced by the pump beam were measured as a function of the pump-probe delay $(\mathrm{t})$. The smallest detectable signal was $\Delta \mathrm{T} / \mathrm{T}=10^{-3}$, 
and the typical excitation density was of the order of $10^{19} \mathrm{~cm}^{-3}$. The experiments were carried out at room temperature in a vacuum chamber with pressure less than $10^{-5}$ torr. Each measurement of $\Delta T / T(t)$ was fit to a convolution of multiple exponential decays and a gaussian representing the temporal resolution of the experiment. From the fit, we extracted the initial differential transmission
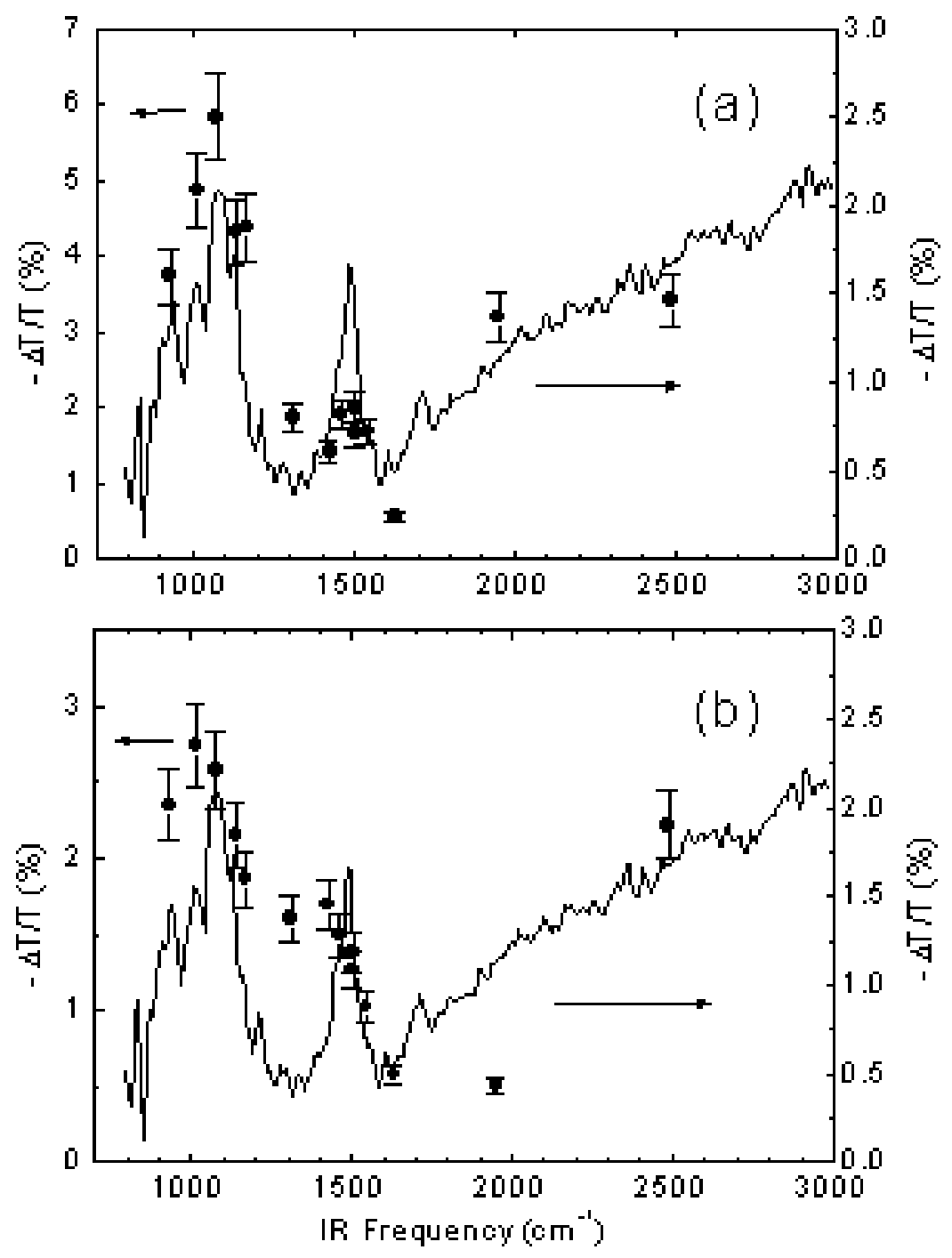

Figure 1. Photoinduced IRAV spectrum for (a) MEH-PPV / $\mathrm{C}_{60}$ blend and (b) pristine MEH-PPV. Solid circles are the ultrafast measurements at " $t=0$ " and $\mathrm{T}=300 \mathrm{~K}$. For comparison, the solid line in both (a) and (b) is the steady-state IRAV spectrum for the MEH-PPV/C 60 blend $(\mathrm{T}=90 \mathrm{~K})$. The pump wavelength was $494 \mathrm{~nm}$ and $514 \mathrm{~nm}$ for the ultrafast and steady-state measurements, respectively.

$\Delta \mathrm{T} / \mathrm{T}(0)$, which is related to the quantum efficiency for charge pair generation $\left(\phi_{\mathrm{ch}}\right)$ by $\Delta \mathrm{T} / \mathrm{T}(0)=2$ $\phi_{\mathrm{ch}} \sigma \mathrm{F}$, where $\mathrm{F}$ is the photon flux absorbed by the sample (photons $/ \mathrm{cm}^{2}$ ) and $\sigma$ is the cross-section for IRAV absorption.

There is direct experimental evidence that neutral bound-state excitations, such as intrachain or interchain excitons, do not contribute to the measured photoinduced IRAV absorption. First, the 
addition of $\mathrm{C}_{60}$ to $\mathrm{MEH}-\mathrm{PPV}$ is known to promote ultrafast electron transfer from the polymer to $\mathrm{C}_{60}$, yielding a positive polaron in the polymer and an electron in $\mathrm{C}_{60}$ [12]. For high enough $\mathrm{C}_{60}$ concentrations, this process has quantum efficiency close to $100 \%$ and leads to nearly complete luminescence quenching and increased PC (with charge collection efficiencies higher than $60 \%$ ) [13]. Previous reports demonstrated that the ultrafast IRAV signal is enhanced by a factor $\sim 3$ when adding $50 \%$ by weight of $\mathrm{C}_{60}$ to $\mathrm{MEH}-\mathrm{PPV}$ [4]. If excitons were contributing to the mid-IR absorption, however, the signal would have been reduced by exciton quenching. From the ratio of IRAV signals in pristine MEH-PPV and the MEH-PPV / $\mathrm{C}_{60}$ blend, the quantum efficiency for charge pair generation in pristine MEH-PPV when pumped at $400 \mathrm{~nm}$ was estimated as $\sim 10 \%$ (assuming the electron transfer efficiency in the blend to be 100\%). Moreover, the different dynamics of the IRAV photoinduced absorption and the stimulated emission in MEH-PPV under identical excitation conditions [14] indicates that excitons cannot be the major contribution to the IRAV signal.

Figure 1 shows the spectra of the IRAV modes obtained with steady-state excitation for the $\mathrm{MEH}-\mathrm{PPV} / \mathrm{C}_{60}$ blend, and with the ultrafast setup for both $\mathrm{MEH}-\mathrm{PPV}$ and the MEH-PPV / $\mathrm{C}_{60}$ blend. The steady-state and ultrafast spectra are in reasonable agreement, with all the spectral features present in both. They are also in good agreement with the data obtained at $\sim 100 \mathrm{ps}$ time resolution [3]. The ultrafast photoinduced IRAV modes demonstrate that polarons are produced in less than $100 \mathrm{fs}$, consistent with the early predictions of Su and Schrieffer [15].

Figure 2 shows the excitation spectrum for ultrafast charge generation for stretch-oriented PPV and for MEH-PPV (not oriented) from the onset of absorption up to $6.2 \mathrm{eV}$ (filled circles). The most striking feature is the weak dependence of $\phi_{c h}$ over such a wide range of photon energies: PPV shows a modest increase above $3.5 \mathrm{eV}$, which is not observed in MEH-PPV. Data obtained from BuEH-PPV (not shown) were very similar to those from MEH-PPV. Figure 2 shows that the product $\sigma \cdot \phi_{\mathrm{ch}}$ is about twice larger in chain-aligned PPV than in MEH-PPV when pumped on the main absorption peak. Although different interchain interactions might play a role [16,17], this is more likely a result of the alignment of the PPV chains in contrast to the random orientation in MEH-PPV. This should enhance $\sigma$ by a factor of two since the IRAV absorption is polarized parallel to the polymer chain. Thus, with $\phi_{\mathrm{ch}} \sim 10 \%$ for MEH-PPV [4], Figure 2(a) shows that $\phi_{\mathrm{ch}} \sim$ $35 \%$ at pump energies above $4 \mathrm{eV}$, implying that under such conditions charged polarons are a very significant fraction of the photoexcitations in the polymer. Indeed, Ruseckas et al. [17] have independently shown that charged polarons are generated within $\sim 100 \mathrm{fs}$ with $\phi_{\mathrm{ch}} \sim 20 \%$ in a polythiophene derivative. They suggested that these charges are inter-chain pairs, with oppositely charged polarons residing on neighboring chains. Although we cannot conclusively address the intra- versus inter- chain nature of the charge pairs, we emphasize they cannot be in a bound state.

It is interesting to compare these results with previous PC measurements in MEH-PPV. The dashed line in Figure 2(b) is the steady-state PC action spectrum taken from Ref. [18], obtained with a sandwich cell configuration. It shows a much more pronounced increase in the ultraviolet than our direct measurements of $\phi_{\mathrm{ch}}$. An even larger increase can be found in Ref. [19]. These PC measurements are, in fact, the only strong experimental support of the notion of strongly bound excitons. Because this strong increase is not observed in the excitation spectrum of $\phi_{\text {ch }}$, we have performed transient and steady-state PC measurements using the more reliable surface electrode geometry [20] on the stretch-aligned PPV film, with both the electric field and light polarization parallel to the polymer chains. The results are also shown in Figure 2(a). In contrast to earlier 


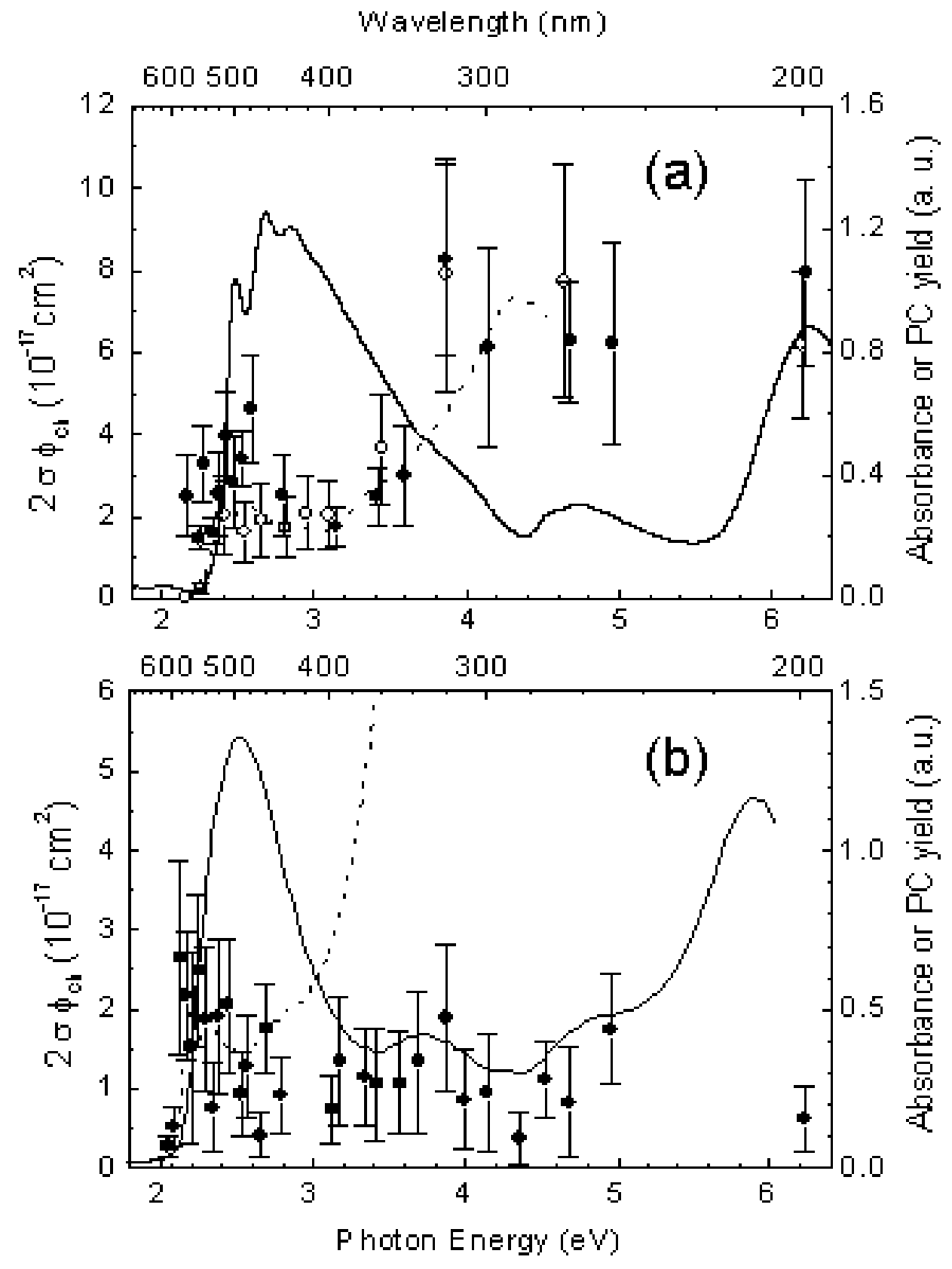

Figure 2. Charge photogeneration excitation spectrum for (a) PPV and (b) pristine MEHPPV. Solid circles are the ultrafast measurements (IR probe at $1100 \mathrm{~cm}^{-1}$ ) and solid lines are the sample absorption spectrum (the spectrum in (a) was taken from Ref. [26]. Dashed lines are steady-state PC action spectra (curve in (b) was taken from Ref. [18]. The empty circles in (a) are transient PC measurements.

reports for related systems, there is good agreement between the ultrafast IRAV and the transient and steady-state PC measurements. The only difference appears at the onset of absorption, where 
the IRAV measurements indicate that charges are generated, but no photocurrent is detected. We conclude that these charges are in disorder-induced localized states that cannot contribute to conductivity, but do generate IRAV absorption. The enhanced charge generation near the onset of $\pi-\pi^{*}$ absorption in MEH-PPV (Figure 2(b)) is consistent with increased disorder in the spin-cast film.
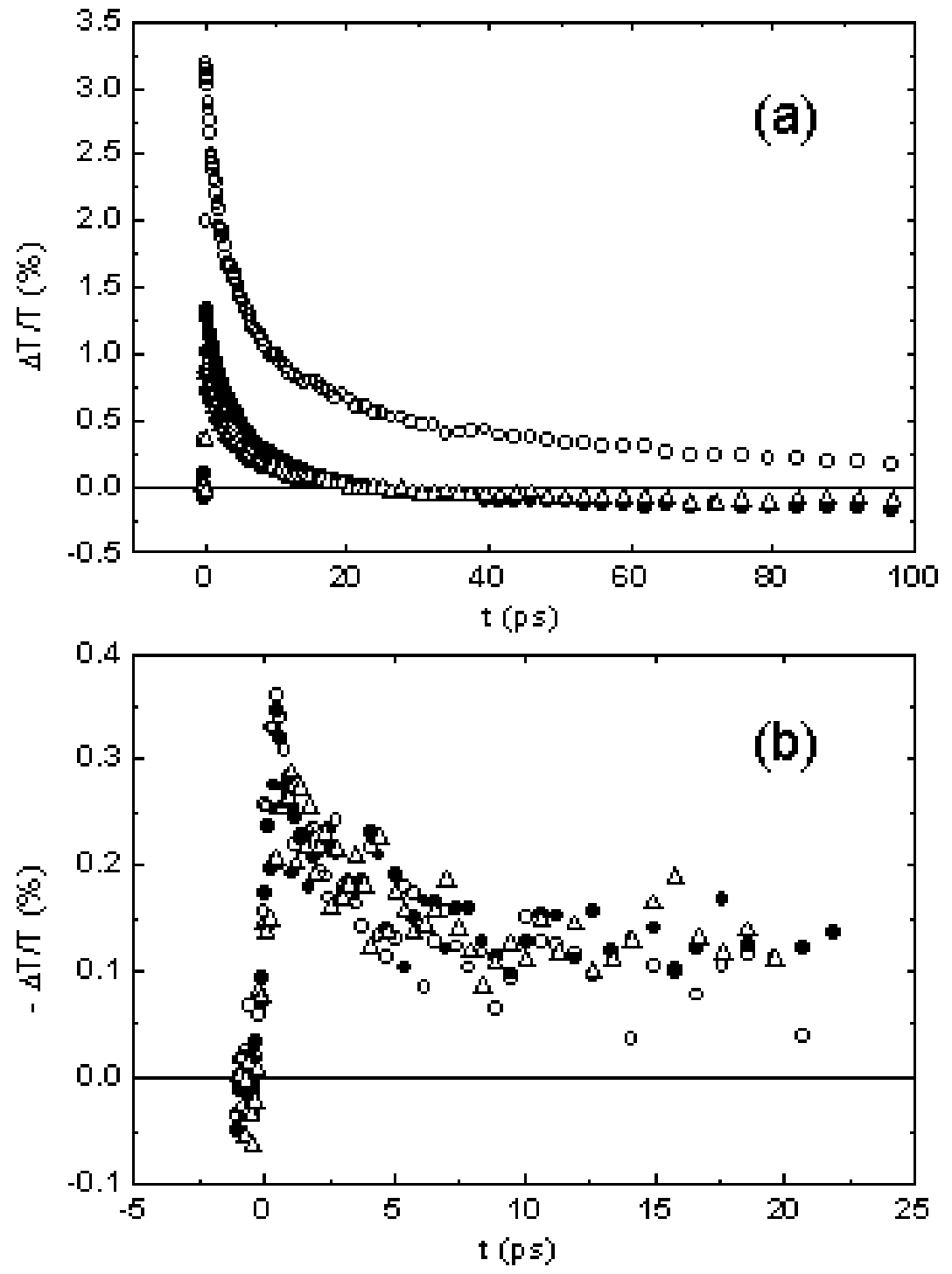

Figure 3. Sequence of three measurements of (a) SE at $633 \mathrm{~nm}$ and (b) IRAV photoinduced absorption at $1100 \mathrm{~cm}^{-1}$, for MEH-PPV pumped at $482 \mathrm{~nm}$. Empty circles are for a pristine sample in vacuum. Solid circles and empty triangles are two consecutive measurements with the sample in air.

The weak wavelength dependence of $\phi_{\mathrm{ch}}$ and the ultrafast nature of charge generation $(<100 \mathrm{fs})$ imply that charged polaron pairs are directly photogenerated at all photon energies above the onset of $\pi-\pi^{*}$ absorption. Furthermore, we have observed a linear dependence on pump intensity for both 
ultrafast IRAV and stimulated emission signals (probing charge and exciton densities, respectively). These results eliminate several indirect charge generation mechanisms proposed in the literature. Since these measurements were performed with no applied electric field on a pristine freestanding film, field-induced exciton dissociation and photoinjection from electrodes are not possible. It has also been suggested that bimolecular exciton annihilation would be responsible for charge generation at high optical excitation densities [21]. However, the linear intensity dependence of the charge density is in disagreement with this notion. Although a strong bimolecular decay of the stimulated emission (SE) is observed at high intensities (initial decay $\sim 2 \mathrm{ps}$ ), the risetime of the photoinduced IRAV signal is always resolution-limited. Therefore, bimolecular decay of excitons is not a significant source of charges. Sequential two-photon excitation of highly energetic excitons has been proposed as a mechanism for ultrafast charge generation at high excitation intensities [22].

However, in order to have a linear intensity dependence of the charge density, this mechanism requires a saturation of the low-energy exciton density, which is not observed in our experiments (data not shown).

Moreover, there is no sign of the resonance expected from the sequential two-photon mechanism as the pump energy is tuned through the absorption band. Furthermore, this mechanism assumes that charge generation is considerably more efficient upon excitation at high photon energies, also in disagreement with the results in Figure 2. Hot-exciton dissociation [23] is still another mechanism proposed to explain charge generation in the presence of electric fields, where phonons emitted upon relaxation of the initial excited state into a bound exciton form a thermal bath that induces exciton dissociation before the vibrational cooling time. It could still lead to ultrafast charge generation in zero electric field, although the charges would eventually recombine into a bound exciton again. However, in order to explain the weak wavelength dependence of $\phi_{\mathrm{ch}}$, its magnitude and the ultrafast charge generation with reasonable parameters, the exciton binding energy has to be small $(<0.1 \mathrm{eV})$.

Finally, the dynamics of the photo-induced IRAV absorption is generally non-exponential and quite short-lived (a typical example is shown in Figure 3(b)) but it depends significantly on the initial excitation density, with larger densities leading to shorter decays. This is indeed what should be expected for charged polaron pair recombination, which happens within tens of picoseconds in the conditions of our experiments.

\section{THE ROLE OF ELECTRON PHOTOEMISSION IN PHOTOCONDUCTIVITY MEASUREMENTS}

It seems that the carrier generation problem has remained controversial, principally because of reports of a dramatic increase in the photocurrent at energies well above the onset of absorption in PPV derivatives $[2,5,19,22]$. In particular, based on the action spectrum of the steady-state photoconductivity in MEH-PPV, which indicated a sharp rise above $\mathrm{hv} \sim 3.5 \mathrm{eV}$, Chandross et al. concluded that the primary excitations in this system are excitons and that the exciton binding energy is $\sim 1 \mathrm{eV}$ [18]. Such a large binding energy would imply localized Frenkel excitons and the importance of geminate recombination.

Recently, Wegewijs et al [24] have demonstrated that photoemission (PE) of electrons may contribute significantly to the photocurrent at high photon energies (photon energies approaching the work function of the semiconducting polymer). As a result, we have measured the excitation profile of the transient photoconductivity in several conjugated polymers over a wide spectral range, from the absorption edge up to $6.2 \mathrm{eV}$. We have identified and separated the distinct 
contribution that arises from true photoconductivity (carrier transport in the bulk of the semiconducting polymer) and from that which arises from the electron PE current. At high light intensities, the PE contribution becomes significant, particularly at photon energies above 3-4 eV. However, when this contribution is quenched by addition of $\mathrm{CO}_{2}+\mathrm{SF}_{6}(90 \%: 10 \%)$ into the sample chamber, the bulk PC is nearly independent of photon energy over the entire spectral range up to $6.2 \mathrm{eV}$. The flat photoconductivity $\left(\sigma_{\mathrm{ph}}\right)$ excitation profile is fully consistent with the excitation profile for the photogeneration of carriers $\left(n_{p h}=n_{e}+n_{h}=2 n_{e}\right)$, as expected since $\sigma_{p h}=e\left(n_{e} \mu_{e}+n_{h} \mu_{h}\right)$, where $\mu_{\mathrm{e}}$ and $\mu_{\mathrm{h}}$ are the mobility of the electron and hole, respectively.

Although the number of electrons ejected from the sample surface (and possibly the Au contacts [25,26]) by photoemission is relatively small (e.g. much smaller than the number of the photoexcited electrons in the bulk), the contribution of the electron PE to the transient photocurrent is significant since the fringe field from the surface contacts can induce a drift velocity that is high compared to the bulk carrier drift velocity [27]. This contribution is expected to increase at higher light intensities and higher photon energies as the probability for electron photoemission increases. As demonstrated in this report, although the light intensity used in steady-state photoconductivity is relatively small compared to that used in the transient PC, when the hv is greater than the work function the PE contribution is significant.

The PPV samples were freestanding films, tensile drawn and stretch-oriented to a draw ratio of $1 / 1_{0}=4$. Samples of the PPV derivatives were in the form of (nonoriented) thin films $(\sim 200 \mathrm{~nm}$ thick) that were spin cast onto alumina substrates. All films were prepared with Au surface contacts in the Auston switch configuration, where the gap between the electrodes (that determines the effective sample length) was approximately $200 \mu \mathrm{m}$ for the samples used for transient PC and about $20 \mu \mathrm{m}$ for the ones used for the steady-state PC.

The separation of the bulk photocurrent from that due to free electrons in vacuum was achieved by comparing the photocurrent response while the sample was kept in modest vacuum (as achieved by mechanical pump that yielded a pressure of $\sim 60$ mTorr) to that obtained with the sample in an environment of a gas mixture consisting of $90 \% \mathrm{CO}_{2}$ and $10 \% \mathrm{SF}_{6}$ at atmospheric pressure. This gas mixture effectively eliminates the free electron contribution, both by impeding the electron motion (mostly by the $\mathrm{CO}_{2}$ molecules) [27,28] and electron capture by the high electron affinity $\mathrm{SF}_{6}$ molecules [29]. Note that the residual gas pressure in the modest vacuum used in our experiments limits the magnitude of the PE contribution. In the photon energy range studied, the optical absorption due to the gas mixture is negligible [30].

The excitation source was an amplified Ti:Sapphire laser system including an optical parametric amplifier (OPA) tunable up to $\sim 4 \mathrm{eV}$, and a system that produces the third and fourth harmonics of the Ti:Sapphire laser fundamental. The laser yields pulses of $\sim 100$ fs duration at 1 $\mathrm{KHz}$ rate. In order to determine the excitation profile, we used a gap between the electrodes on the Auston switch of $0.2 \mathrm{~mm}$ with light intensity of $\mathrm{I}=100 \mu \mathrm{J} / \mathrm{cm}^{2}$ per pulse, and bias field of $\mathrm{F}=20 \mathrm{KV} / \mathrm{cm}$ that were kept constant at all photon energies. The photocurrent values were normalized to a constant photon flux of $3.5 \times 10^{14}$ photons $/ \mathrm{cm}^{2}$ per pulse, taking into account the distinct dependence of the PC on light intensity measured at each photon energy. Note that in these experiments, we used a $200 \mu \mathrm{m}$ gap rather than a $20 \mu \mathrm{m}$ gap and higher light intensities than typically used in routine transient photoconductivity measurements in our laboratory. The steady-state PC was measured by conventional modulation technique, whereby the light of a Xe lamp was dispersed by a monochromator and chopped, while the photocurrent was measured by a lock-in amplifier. 
We first verified that the contribution of the PE from the underlying alumina substrates was negligible for all samples studied. In addition, we established that exposure of the samples to the gas mixture does not affect the dark conductivity, implying that the quenching of the PE by the gas mixture does not originate from chemical doping of the polymers. In the range of the bias field used of $\mathrm{F}<25 \mathrm{KV} / \mathrm{cm}$, both the bulk photocurrent and that due to the PE were linearly proportional to $\mathrm{F}$.

Figure 4 shows the excitation profile of the photocurrent in MEH-PPV measured in vacuum and in an environment of $\mathrm{CO}_{2}+\mathrm{SF}_{6}(90 \%: 10 \%)$; similar results for PPV are shown in the inset. As determined from the photocurrent response obtained with the sample in the environment of the gas mixture that quenches the PE contribution, the bulk peak transient photoconductivity in PPV and in all the PPV derivatives studied is essentially independent of photon energy, up to 6.2 $\mathrm{eV}$. As indicated from the inset of Figure 4, the contribution of the PE to the photocurrent in PPV is negligible at photon energies below $\sim 3 \mathrm{eV}$, but becomes significant at higher photon energies

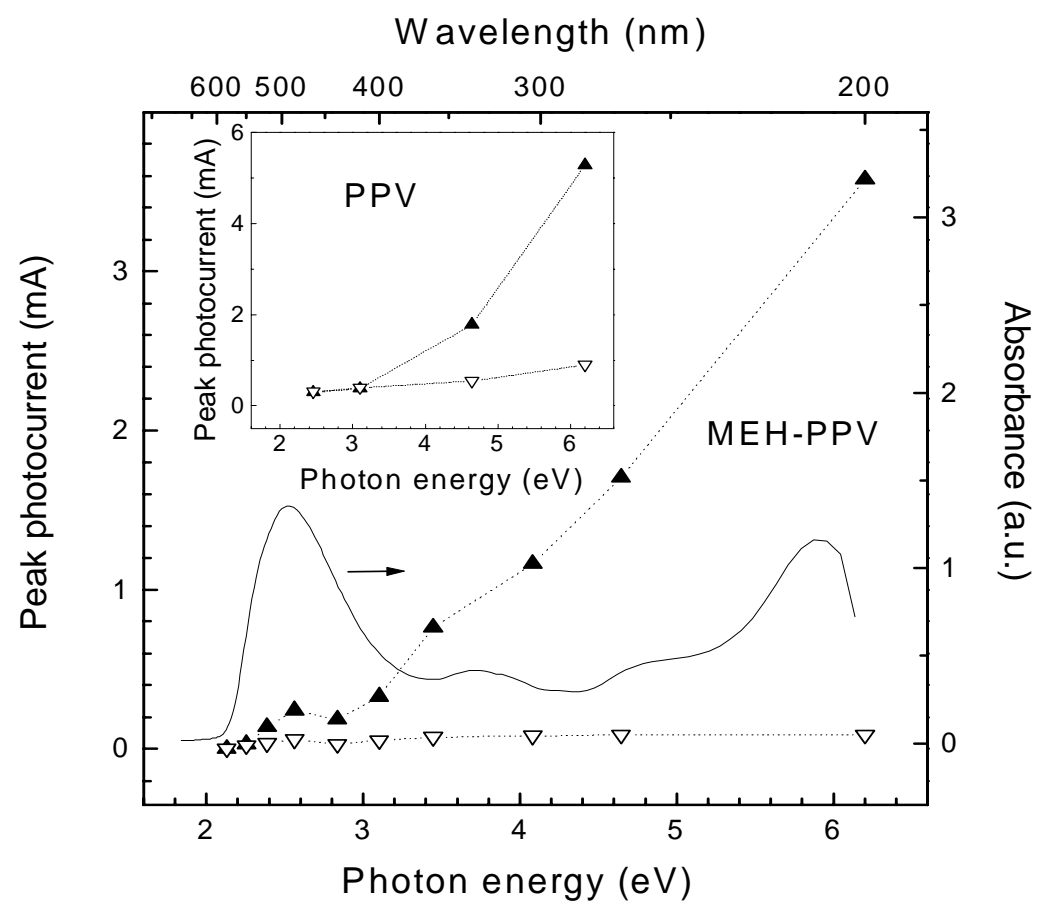

Figure 4. Excitation profile of the peak transient PC in MEH-PPV as measured while the sample was in vacuum $(\boldsymbol{\Delta})$ and in an environment of $\mathrm{CO}_{2}+\mathrm{SF}_{6}(90 \%: 10 \%)$ gas mixture at atmospheric pressure $(\nabla)$, normalized to a constant photon flux of $3.5 \times 10^{14}$ photons $/ \mathrm{cm}^{2}$ per pulse and $\mathrm{F}=20 \mathrm{KV} / \mathrm{cm}$; the absorption profile of MEH-PPV is represented by the smooth line; the inset shows similar data for PPV.

and eventually dominates the photocurrent response. In contrast, MEH-PPV exhibits a PE contribution at all photon energies above the absorption edge.

Measurements on samples with a smaller gap between electrodes, $\sim 20 \mu \mathrm{m}$, using smaller light intensity and higher external field (as typically used in our transient PC measurements), yield a smaller PE contribution to the photocurrent signal than that due to the bulk PC. For example, even at a photon energy of $4.6 \mathrm{eV}$, the PE contribution measured in PPV sample at I= 20 
$\mu \mathrm{J} / \mathrm{cm}^{2} /$ pulse was a fraction $(\sim 0.5)$ of the bulk transient PC whereas at higher light intensity (used for the $0.2 \mathrm{~mm}$ gap) it was greater than the bulk PC by a factor of 20 (as indicated in Figure 4).

The independence of the bulk PC on photon energy is in disagreement with Frenkel excitons as the photoexcitations [2,31]. According to the Onsager model of geminate recombination $[32,33]$, the higher the photon energy, the larger is the initial distance between the thermalized geminate carriers and the higher the probability of their eventual separation into mobile carriers. Thus, although both $\mathrm{n}_{\mathrm{ph}}$ and the photoconductivity are predicted by the Onsager model to increase with photon energy, both are measured to be essentially independent of photon energy.

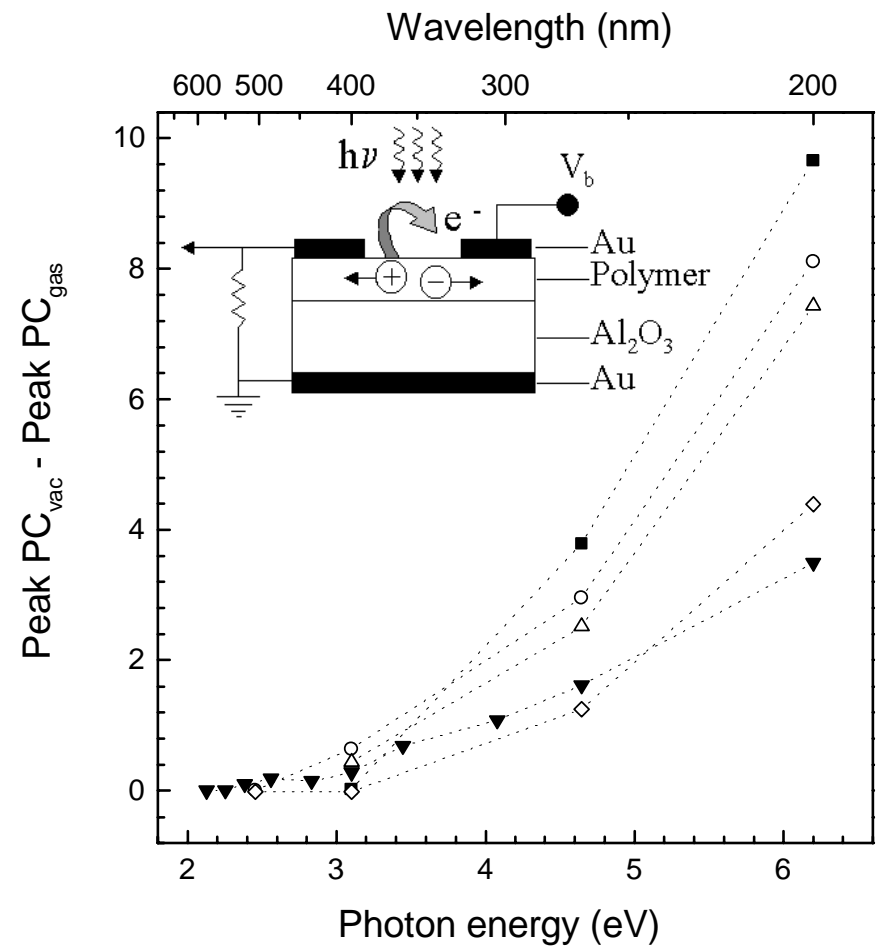

Figure 5. Excitation profile of the PE contribution to the transport in a series of PPV derivatives: - BCHA-PPV, ०- BUEH-PPV, $\triangle$ - BEH-PPV, $\nabla$ - MEH-PPV, $\diamond$ - PPV; the inset shows schematically the sample measuring configuration.

The results in Figure 4 are also inconsistent with the model developed by Arkhipov et al. [34,35], where the carrier generation was proposed to result from hot exciton dissociation. According to Arkhipov et al, the probability of carrier photogeneration depends on the magnitude of excess photon energy above the singlet exciton. This excess energy dissipates into the vibrational thermal bath that is considered as the main source of energy required for the geminate charges to escape recombination (i.e. to escape the potential well formed by superposition of the Coulomb and external field). The observation that the bulk transient photoconductivity is independent of photon energy is inconsistent with the strong dependence on photon energy predicted by Arkhipov et al.

Figure 5 shows the excitation profile of the PE contribution to the transient photocurrent response for all the polymers studied, as deduced from the difference between the photocurrent 
measured in vacuum and that measured with the sample immersed in the gas mixture. The data indicate a tendency of higher PE and smaller bulk contributions to the photocurrent in polymer derivatives with bulky side groups such as BCHA-PPV and BUEH-PPV.

Figure 6 shows the intensity dependence of the PE contribution to the photoconductive response in MEH-PPV at various photon energies. The photocurrent contribution from PE is proportional to $\mathrm{I}^{\mathrm{n}}$. The $\mathrm{PC} \sim \mathrm{I}^{3}$ dependence observed at $2.4 \mathrm{eV}$ indicates that the PE arises from a third-order process (e.g., three photon absorption), whereas at $6.2 \mathrm{eV}$, the PC $\sim$ I, indicating a first-order process. Thus, at $6.2 \mathrm{eV}$, the photon energy exceeds the threshold energy for photoemission, in agreement with previous analysis that concluded that the single photon PE threshold for PPV occurs at $5.2 \mathrm{eV}$ [36]. Table 1 summarizes the values of the power law exponent, $\mathrm{n}$, deduced for the different PPV derivatives at various photon energies.

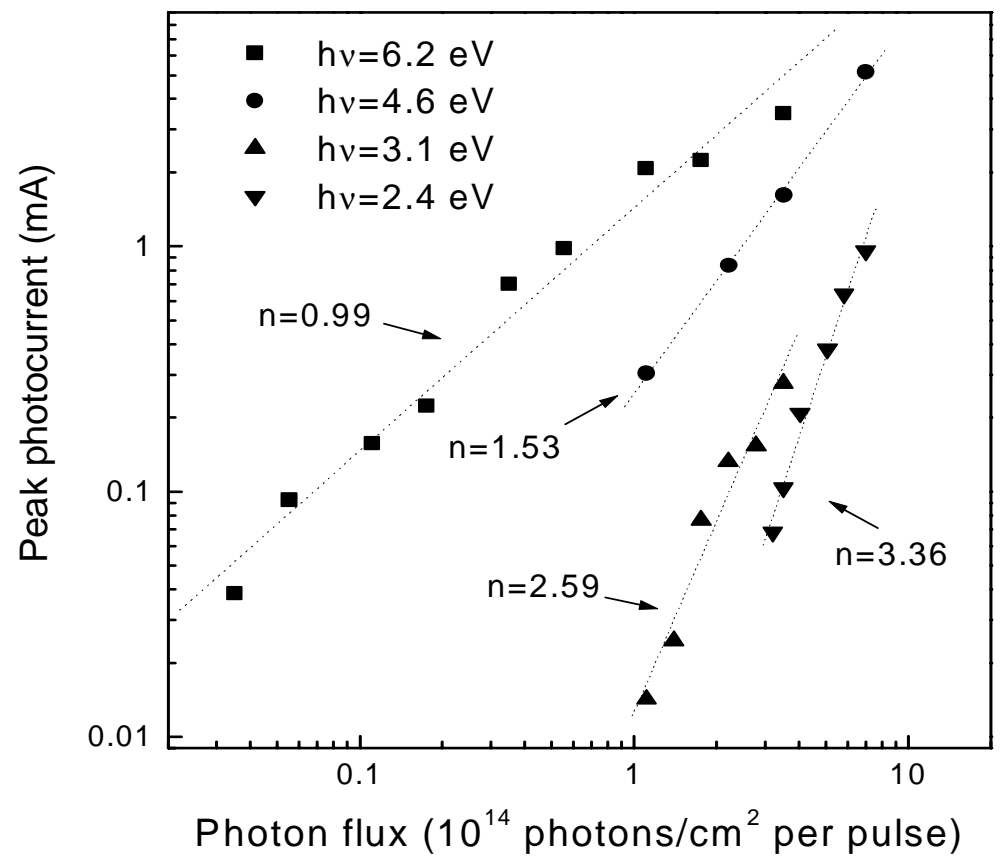

Figure 6. The dependence of the PE contribution to the photocurrent on photon flux in MEH-PPV at various photon energies; the PE contribution is proportional to $\mathrm{I}^{\mathrm{n}}$; the indicated values of $n$ were obtained from fitting of the PE contribution; the different power laws are represented by the dotted lines.

For all the PPV derivatives, the bulk transient PC was linearly proportional to I at all photon energies. The results for oriented PPV (see Figure 4,5 and Table 1) were deduced from measurements with light polarization perpendicular to both the chain axis and the bias field direction. At all the photon energies studied, the data obtained with light polarized perpendicular to the chain axis indicate slightly higher photoconductive response than that measured with polarized parallel to the chain axis. 
Figure 7 displays the excitation profile of the steady-state photocurrent in MEH-PPV as measured with an external field of $\mathrm{F}=20 \mathrm{KV} / \mathrm{cm}$ while the sample was in vacuum and in an environment of $\mathrm{CO}_{2}+\mathrm{SF}_{6}(90 \%: 10 \%)$ gas mixture at atmospheric pressure, while the inset shows similar data for BEH-PPV.

The data indicate a weak dependence of the bulk PC on photon energy, in agreement with the

Table 1. The order of the nonlinear PE excitation process, $n$, as determined from fitting the intensity dependence of the PE contribution to the photocurrent at different photon energies, for the various PPV derivatives.

\begin{tabular}{cccccc}
\hline \hline Energy $(\mathrm{eV})$ & PPV & MEH-PPV & BEH-PPV & BUEH-PPV & BCHA-PPV \\
& & & & & \\
\hline & 1.13 & 0.99 & 1.31 & 1.12 & 1.09 \\
4.6 & 1.24 & 1.53 & 1.83 & 1.84 & 1.99 \\
3.1 & & 2.59 & 2.67 & 3.02 & \\
2.4 & & 3.36 & & & \\
\hline
\end{tabular}

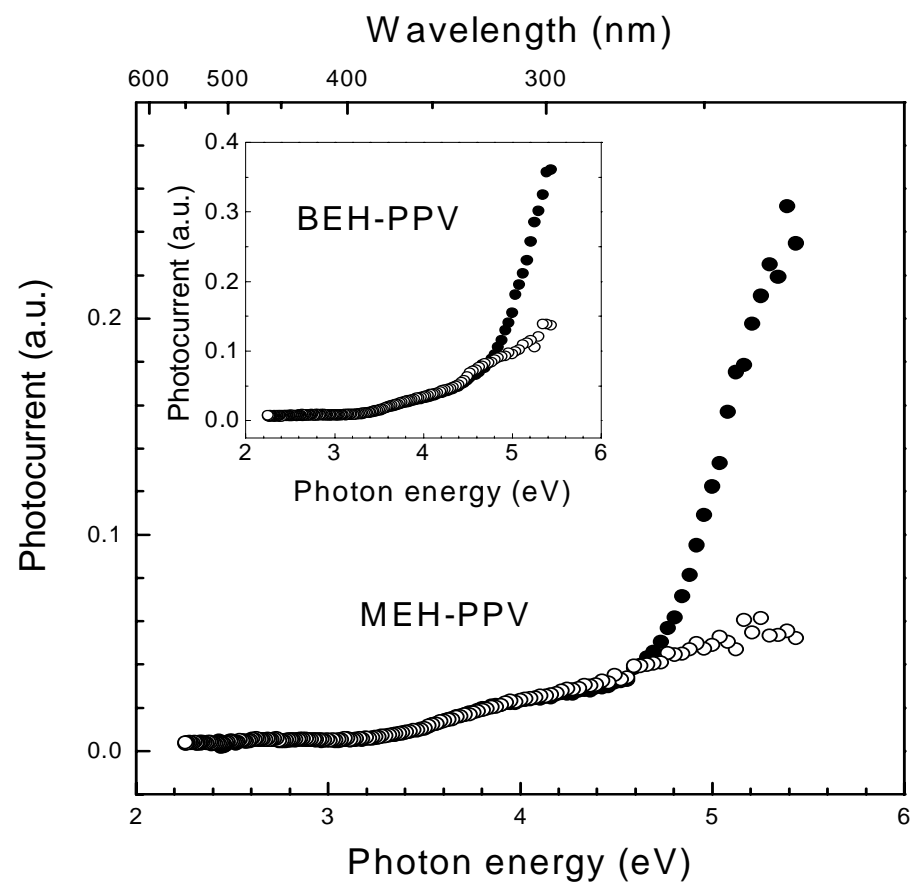

Figure 7. Excitation profile of the steady-state photocurrent in MEH-PPV as measured while the sample was in vacuum $(\bullet)$ and in an environment of CO2 + SF6 (90\%:10\%) gas mixture at atmospheric pressure $(\circ)$; the data were collected with an external field of $\mathrm{F}=20 \mathrm{KV} / \mathrm{cm}$ and normalized to constant light intensity; the inset shows similar data for BEH-PPV. 
Transient PC data as well as with the IRAV absorption measurements, and a rise in the apparent PC that results from the PE contribution above $\sim 4.7 \mathrm{eV}$. Although the light intensity in steadystate PC is much smaller than that used for transient PC measurements, (and thus light absorption via nonlinear processes is relatively small) the experiments indicate that for hv greater than the work function, the PE contribution to the steady-state photocurrent can be significant.

\section{CONCLUSIONS}

In conclusion, the photoinduced IRAV measurements have shown that polarons are generated in conjugated polymers within $\sim 100$ fs with appreciable quantum efficiencies $(\sim 10 \%)$ that are only weakly dependent on the excitation wavelength. The data are not consistent with indirect charge generation by excitons of large binding energy. It is clear that both excitons and polarons are independently generated even at times less than $100 \mathrm{fs}$. The mechanism responsible for this intrinsic ultrafast branching of the photoexcitations into neutral excitons and charged polarons remains to be elucidated.

We have measured the excitation profile of the transient and steady-state photocurrent in various derivatives of PPV. We have detected and characterized a contribution to the transient and steady-state photocurrent due to electron photoemission. After quenching the PE contribution, the transient photoconductivity is nearly independent of the excitation energy (up to $6.2 \mathrm{eV}$ ) in all the PPV derivatives.

The photoconductivity data and the $\mathrm{n}_{\mathrm{ph}}$ data, obtained independently from the ultrafast photoinduced IRAV mode absorption, indicate a single threshold for the ultrafast photogeneration of charged carriers. Moreover, the single threshold is spectrally close to the onset of optical absorption. The absence of a second threshold in the excitation profile for the photoconductivity and $\mathrm{n}_{\mathrm{ph}}$ implies that the lowest energy absorption results from the lowest $\pi-\pi^{*}$ interband transition and that the excition binding energy is small. High resolution measurements of the excitation profile of the photoconductivity as a function of the electric field near the onset of interband absorption have identified the exciton in PPV through field ionization of the electron-hole bound state. In PPV, the exciton binding energy is approx. $60 \mathrm{meV}$.

Finally, for photon energies higher than the onset of the lowest $\pi-\pi^{*}$ interband transition, carrier photogeneration in PPV and its derivatives is nearly independent of photon energy, temperature, and external field. Thus, the photophysics of semiconducting polymers is similar to that of direct gap inorganic semiconductors.

\section{ACKNOWLEDGEMENTS}

The authors thank Dr. G. Bahir for preparing the contacts on the GaAs sample and for important discussions, and C. Soci for assistant with the activation energy measurements. The research was supported by NSF grants DMR 0096820.

\section{REFERENCES}

1. M.D. McGehee e. k. Miller, D. Moses, A. J. Heeger, in P. Bernier, (ed.), "Advances in Synthetic Metals", Elsevier, Lausanne, 1999, p. 98.

2. D. Moses in "The Nature of Photoexcitations in Conjugated Polymers", edited by N.S. Sariciftci, (ed.), (World Scientific, Singapore, 1997).

3. U. Mizrahi, I. Shtrichman, D. Gershoni, E. Ehrenfreund, Synth. Met. 102, 1182 (1998). 
4. D. Moses, A. Dogariu, A.J. Heeger, Phys. Rev. B 61, 9373 (2000).

5. S. Barth and H. Bässler, Phys. Rev. Lett. 79, 4445 (1997).

6. A. J. Heeger, S. Kivelson, J. R. Schrieffer, W.P. Su, Rev. Mod. Phys. 60, 781 (1988).

7. N. Kirova, S. Brazovskii, A. R. Bishop, Synth. Met. 100, 29 (1999).

8. T. K. Däubler, V. Cimrova, S. Pfeiffer, H. Horhold, D. Neher, D. Adv. Mater. 11, 1274 (1999).

9. C. R. Fincher, M. Ozaki, A. J. Heeger, A. G. MacDiarmit, Phys. Rev. B 19, 4140 (1979).

10. B. Horowitz, Solid State Commun. 41, 729 (1982).

11. Z. G. Soos, A. Girlando, A. Painelli, Molecular Cryst. And Liq. Cryst. 256, 711 (1994).

12. N. S. Sariciftici, L. Smilowitz, A. J. Heeger, F. Wudl, Science 258, 1474 (1992).

13. G. Yu, J. Gau, J. C. Hummelen, F. Wudl, A. J. Heeger, Science 270, 1789 (1995).

14. Details will be published elsewhere.

15. W. P. Su and J. R. Schrieffer, Proc. Natl. Acad. Sci. USA 77, 5626 (1980).

16. Z. G. Soos, G. W. Haiden, A. Girlando, A. Painelli J. Chem. Phys. 100, 7144 (1994).

17. A. Ruseckas, M. Theander, M. R. Andersson, M. Svensson, M. Prato, O. Inganas, V. Sundstrom, Chem. Phys. Lett. 322, 136 (2000).

18. M. Chandross, S. Mazumdar, S. Jeglinski, X. Wei, Z. V. Vardeny, E. W. Kwock, T. M. Miller, Phys. Rev. B 50, 14702 (1994).

19. A. Köhler, D. A. Santos, D. Beljonne, Z. Shuai, J. -L. Bredas, A. B. Holmes,

A. Kraus, K. Mullen, R. H. Friend, Nature 392, 903 (1998).

20. D. Moses, Phys. Rev. B 53, 4462 (1996).

21. G. J. Denton, N. Tessler, M.A. Stevens, R.H. Friend, Synth. Met. 102, 1008 (1999).

22. C. Silva, M.A. Stevens, D.M. Russell, S. Setayesh, K. Mullen, R.H. Friend, Synth. Met. 116, 9 (2001).

23. V. I. Arkhipov, H. Bassler, E. O. Gobel, Phys. Rev. Lett. 82, 1321 (1999).

24. B. R. Wegewijs, G. Dicker, J. Piris, A.A. Garcia, M.P. De Haas, J.M. Warman, J.M Chem. Phys. Lett. 332, 79 (2000).

25. J. P. Girardeau-Montaut and C. Girardeau-Montaut, Appl. Phys. Lett. 62, 426 (1993).

26. N. A. Papadoggianis and S. D. Moustaizis, J. Phys. D: Appl. Phys. 34, 499 (2001).

27. Y. Itikawa, Phys. Fluids 16, 831 (1973).

28. R. D. Hake, A. V. P. Jr., and A. V. Phelps, Phys. Rev. 158, 70 (1967).

29. F. C. Fehsenfeld, J. Chem. Phys. 53, 2000 (1970).

30. F. Bastien, P.A. Chatterton, E. Marode, J.L. Moruzzi, J. Phys. D: Appl. Phys. 18, 1327 (1985).

31. P. Miranda, D. Moses, and A. J. Heeger, Phys. Rev. B, Rapid Comm. 64 (8), 81201 (2001).

32. M. Pope and C. E. Swenberg, Electronic Processes in Organic Crystals (Oxford University press, New York, 1982).

33. R. C. Enck and G. Pfister, in "Photoconductivity and Related Phenomena", J. Mort and D. M. Pai, Eds., (Elsevier Scientific Publications, New York, 1976).

34. V. I. Arkhipov, E. V. Emelianova, and H. Bassler, Phys. Rev. Lett. 82, 1321 (1999).

35. V. I. Arkhipov, E. V. Emelianova, and H. Bassler, Chem. Phys. Lett. 340, 517 (2001).

36. W. R. Salaneck, R. H. Friend, and J. L. Bredae, Phys. Reports 319, 231 (1999). 